\title{
SEAMLESS COMMUNICATION AND ACCESS TO INFORMATION FOR MOBILE USERS IN A WIRELESS ENVIRONMENT
}

\author{
Golha Sharifi, Julita Vassileva and Ralph Deters \\ University of Saskatchewan, Computer Science Department, \\ 57 Campus Drive, Saskatoon, Saskatchewan S7N 5A9, Canada Phone:+1 306 966-4886,Fax:+1 306 966-4884 \\ Email: \{gos787,jiv,ralph\}@cs.usask.ca
}

Keywords: $\quad$ Mobile Devices, Agents

\begin{abstract}
Providing mobile workers with mobile devices such as a Compaq iPaq with a CDPD card can support them in retrieving information from centralized information systems. More specifically, mobile devices can enable mobile users to make notifications for schedule changes and add new data into the information system. In addition these devices can facilitate group communication nytime and anywhere. This paper presents different ways of providing non-critical information in a timely fashion for nomadic users of mobile devices using a wireless network. A distributed application prototype to support nomadic users is proposed, and a simulated environment is used to evaluate the prototype. Since solutions for seamless access are highly domain specific, the study involves homecare workers at Saskatoon District Health (SDH). By keeping track of the users' current context (time, location etc.) and a user task model, it is possible to predict the information needs of mobile users and to provide context dependent adaptation of both the content and the functionality. Moreover, to avoid interrupts in the user's interaction with the main information sources, methods for mobile transactions management using agent-based smart proxies that buffer, delay or pre-fetch information/data are introduced.
\end{abstract}

\section{INTRODUCTION}

The development of wireless technology and the commercial success of small screen appliances, especially cellular phones and Personal Digital Assistants (PDAs) have advanced the development of mobile and ubiquitous computing (Weiser, 1991). Mobile and ubiquitous computing allows nomadic users to remain "connected" without depending on a wired infrastructure.

Nomadic workers who use mobile and small screen devices often need access to existing information services to enable them to browse multimedia information anytime and anywhere with ease. Nomadic workers in specific domains who might require these services include healthcare, emergency services, sales, and education. However, a major problem for the nomadic user using a thin client is the varying quality of the connection. Though connections remain fairly stable and good in areas close to network stations, for instance, Cellular Digital Packet Data (CDPD) towers, it is not uncommon to have zones of low connectivity or even no connection due to physical structures, for example, high concrete buildings blocking the signals.

We have investigated different ways of enabling seamless access of non-critical information for nomadic users. In this paper we focus on the needs of homecare workers of the Saskatoon District Health (SDH). The rest of the paper is organized as follows: in the next section the domain for this study and the current problems faced by mobile users are introduced; next, the general approach to solving these problems is described, which is explained in more detail in the third, fourth and fifth sections; the sixth section outlines an evaluation procedure; a comparison with other work is presented in the 
seventh section; the final section concludes the paper.

\section{RESEARCH DOMAIN}

The SDH was chosen as a result of an ongoing collaboration between Sasktel, TR-Labs and the University of Saskatchewan. Currently, the focus of this research is on the use of resource rich devices, like the Compaq iPaq and the CDPD network to support homecare workers in SDH.

Our research focuses on the problems of nomadic homecare workers in SDH. In SDH each patient has a team of homecare workers assigned to him or her, which consists of nurses, physiotherapists, home health aides, social workers, and dieticians responsible for looking after the patient. Each team meets on a periodic basis to discuss and coordinate necessary treatment for an individual patient. Tasks and duties are divided among the homecare workers based on skills and available time slots. The relation between patients and homecare workers can be described as M:N.

Though each of the homecare team members has different tasks and responsibilities, they all have one task in common: treatment of a patient. Homecare workers visit their patients in their homes. The offices of the homecare workers are usually scattered throughout different buildings and maintain separate scheduling and information systems. To date there has been no centralized information system for their use, which limits their ability to share and exchange information regarding schedules and patients' medical records.

Homecare workers provide health care services in different district areas in SDH. The wireless network's bandwidth they use is low ( $<1$ megabits per second (Mbps)) and varies significantly from one area to another. Currently, Saskatoon mobile homecare workers do not have access to patients' information outside the office, i.e. there is no available remote access to data files, such as health records and daily schedules.

A preliminary study was conducted on the usability of the electronic health records system for homecare at the SDH (Pinelle and Gutwin, 2001). The study describes the way that groups of homecare workers care for a patient and identifies the existing problems. Results from this study suggest that there are several obstacles for communication between the members of a group, which have led to difficulties in making changes in the treatment of patients, coordination among homecare workers, and the scheduling of patient visits. These difficulties are also observed in accessing information while the groups of homecare workers are mobile. In order to address these problems two approaches are proposed: increasing awareness and supporting communication.

Following the recommendations given (Pinelle and Gutwin, 2001), we would like to create a system that supports the access to information and the communication between homecare workers. Because of data-security and safety reasons, we decided not to focus on core health care data but on ways to improve the availability of non-critical information, such as scheduling information and personal patient data, e.g., address and phone number. Access to the patient medical record data requires, according to the general regulation, permission from the patients, which is hard to obtain.

In SDH, homecare workers work in a variety of contexts, such as in the office, on the road, and at the patient's home. When an office scheduler or a homecare worker makes a schedule, she must have knowledge of the other team members' schedules in order to avoid conflicts in visiting the same patient. However, since there is no access to a centralized scheduling system, it is difficult to coordinate schedule changes and take into account new data regarding the patient. For instance, if a homecare worker visits a patient and notices that the patient's condition has worsened, ideally, s/he would immediately enter this information into the patient's record so that the other team members of the group can access the information and perhaps revise their schedules. However, this is not possible due to absence of centralized data storage. We classify the problems encountered by the homecare workers in two categories. The first category has to do with lack of efficient communication between homecare workers, and the second is related to the inefficient system of accessing and updating information.

Providing homecare personnel with mobile devices e.g. iPaq and access to the wireless network based on CDPD will allow them to retrieve information, receive and send notification of schedule changes, update the data/information system and communicate with other workers. Mobile devices come in varieties of types and capabilities. For instance, small screen computing devices differ in their features and abilities compared to desktop computers. Their most notable limitations are their smaller screen size, less powerful processors, less main memory and limited bandwidth because of the wireless network. To ensure scalability, performance, and speed, it is necessary to develop tools and techniques to compensate for these limitations.

In this paper we introduce tools and techniques that enable content and presentation adaptation to improve the access of workers to information/data. 
In particular, we have introduced the use of proxies that create the illusion of a seamless connection. Moreover, the adaptation is based on user, task, and context models. Further, to ensure that the users have the illusion of seamless access, intelligent agent-based (Jennings et al., 1998) proxies are used, which can delay write operations, pre-fetch data and cache data, as well as use the task, context, and user models to decide when and how to cache, pre-fetch or delay.

\section{GENERAL APPROACH}

In order to address these problems, we propose combining techniques from agent technologies, distributed database transaction management, and user and task modeling. One of the goals is to ensure seamless access to the information despite the frequent interruptions in the connection caused by the mobility of the homecare workers and the unequal coverage of the area. The interface has to be designed for small wireless devices allowing homecare workers to conveniently perform typical operations, including the following:

- Retrieving information from the system (downloading files)

- Entering new data into the health records (uploading/updating files)

- Accessing the schedule (reading schedules and making appointments)

- Communicating with other members of the home care division (sending/receiving messages)

It has been noted (Vassileva, 1996) that healthcare and homecare workers have typical tasks, which have standard information needs, e.g., the task of visiting a patient requires information regarding the patient's address. Therefore, it is possible to (1) predict what kind of information will be needed by the homecare worker using her schedule and (2) prefetch and/or adapt information appropriate for the task and (3) present it according to the user's preferences and the limitations of the device on which the information is going to be viewed. More specifically, the techniques proposed to achieve the goals stated in the previous section are listed below:

The use of agents: we use agents to give the users the impression of seamless connection. Agents hide the changes in the network bandwidth from the user by providing the needed information for the users before their information needs arise by the means of task, user, and context modeling and adaptation techniques.

The identification of read/write operations: To ensure data consistency, we identified two different kinds of read and write operations in the
SDH domain. The first kind is reading or writing to data types needing a lock which prevents users from accessing and/or modifying the data simultaneously (e.g. a schedule); the second kind is information that will not be updated simultaneously and therefore does not require a lock (e.g. a patient's record).

Providing only necessary functionalities: When the context (time, location) and the current task of the user is known, the interface can provide only the functionalities needed by the user to perform the task in the specific context. In this way, the interface can be adapted to the constraint of the small device.

\section{USER AND TASK MODELING}

The combination of techniques described in the previous section is integrated in an application architecture called "Mobi-Timar" (Farsi for "mobile caregiver"), which has an interface for homecare workers, an agent middleware and a server side. The following assumptions are made to help us undertake the design:

- There is a centralized schedule for all homecare workers.

- The mobile devices are resource-rich, e.g., have at least 64 megabytes (MB) RAM, a wireless connection with a bandwidth of at least 19.2 kilobits per second (kbps), and the ability to run third party applications.

- Patient and schedule -related data is stored in a centralized repository.

- The wireless network coverage in the area has varying but known bandwidth and latency.

The next sections describe the main components of the Mobi-Timar architecture.

\subsection{User Model}

In the SDH, the homecare workers can be classified based on their profession as nurses, physiotherapists, social workers, home aide workers and dieticians. Therefore, the user models are based on the stereotype approach, as there are stereotypes corresponding to the professions of the homecare workers. There is a general stereotype for "homecare workers" which has subclass stereotypes corresponding to the professions. The stereotype user model is retrieved and initialized when the user logs in for the first time (she has to enter her profession). The stereotype user model does not change over time i.e., it is a long-term model, since the profession of a homecare worker remains the same. The individual user models are instances of the stereotype user classes containing specific 
individual preferences that may change over time, for example, "personal info", "Chief" or "expert" in Figure 2.

Figure 1 shows an example of the stereotype hierarchy. There are two different relations in this hierarchy. One relation is the inheritance between a subclass stereotype and the general stereotype, e.g., all nurses are homecare workers (see Figure 1). The other relationship that is shown in the stereotype hierarchy is the relation between a type of profes sion referred to as "user class" and its task hierarchies, e.g. the user class "Nurse" has a nurse task hierarchy.

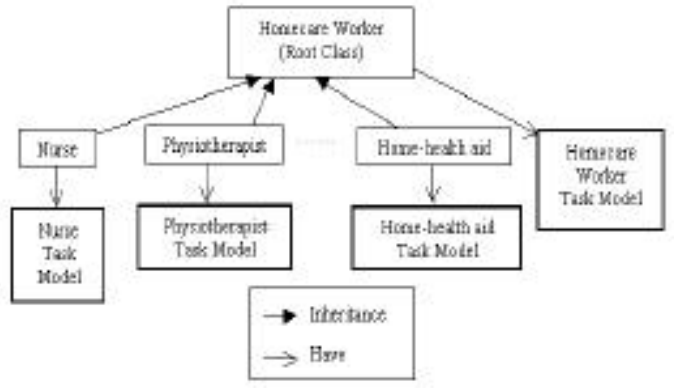

Figure 1: A Stereotype Hierarchy

The individual user model extends the stereotype of the particular user class with the personal information, preferences, rank and the experience of the user. Figure 2 shows an example of an individual user model and illustrates the relationship between this model and the stereotype user model.

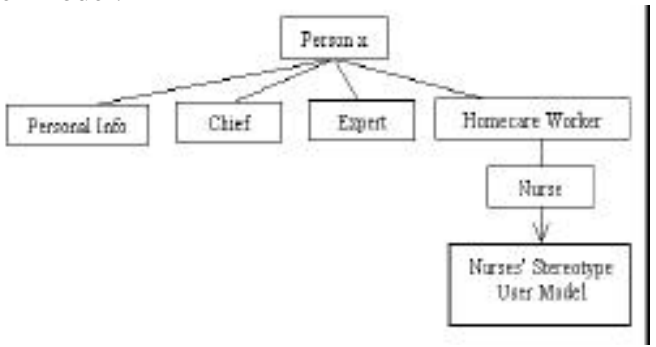

Figure 2: An Example of Individual User Model

\subsection{Task Model}

In SDH each class of users has to perform standardized tasks. Each task in this domain needs specific types of information to be successfully performed, and the information needs of tasks typically do not vary over time. The task hierarchies for each profession contain all the tasks that can be performed by that class of users. Figure 3 shows the task hierarchy for the user class "Nurse."

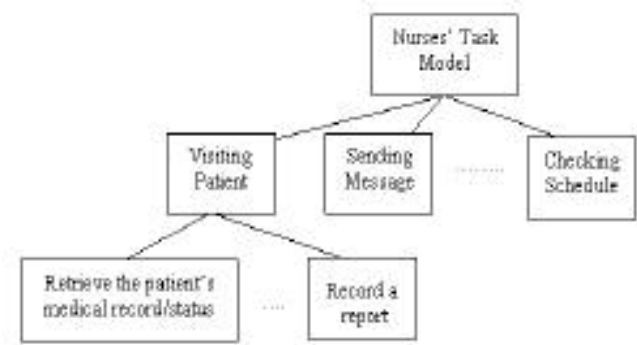

Figure 3: The Task Hierarchy of Nurses Stereotype Class

A detailed view of one task from this task model is shown in Figure 4. The task model is designed hierarchically, and each task is decomposed to subtasks (see Figures 3 and 4). Some of the subtasks are restricted to certain user classes. For the task "Visiting patient" in Figure 4, if the user is a nurse, he needs to perform the following three tasks sequentially:

- Retrieve the patient's medical record

- Retrieve the patient's personal information

- Record the report.

However, if the user is a home-health aide, she will perform only the latter two subtasks. The reason for having task hierarchies defined separately from user task hierarchies is that there can be more than one class of users performing this task. Depending on the user class, different subtasks and different information may be relevant. For example, when a nurse and physiotherapist perform the same task, i.e. retrieving a patient's record, the information that is provided to them is different. Again, depending on the users' profession, access rights to different information are given in each task. For example, in Figure 4, if the user is in the nurse class, he has the right to access the patient's medical record while other classes of users (e.g. social aide workers) do not have access to that information. The lower levels of the task model contain the detailed decompositions for each task and the information needed to perform each subtask. The description of each subtask includes information, such as the users' class and context in which the task can be performed.

As can be seen from Figure 4, each successive level in the task model differentiates subtasks based on either user type or context parameters. At the second level in Figure 4, the task hierarchy is user profession-specific because each task can be carried out differently and can provide different information based on the user's class. In the second level task decomposition takes place. At the third level in Figure 4, the subtasks are organized based on network bandwidth because different available bandwidth allows the task to be carried out differently. Depending on the current bandwidth 


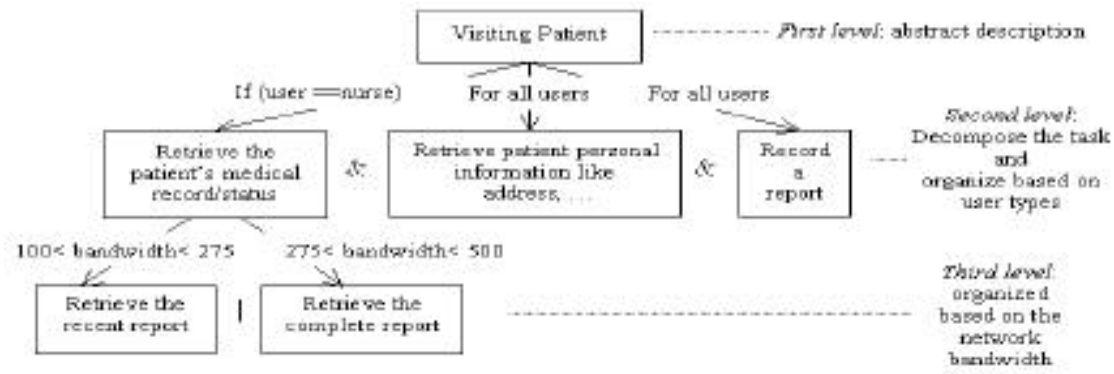

Figure 4: Task Hierarchy for Task "Visiting Patient"

availability, only one of the subtasks will be performed. The changes of task structures and the required information for tasks are regulated and can be changed only by the system administrator.

\subsection{Schedule}

The schedule is used to predict the task that a user will perform and the context (time, location), so the needed information can be pre-fetched according to the task model. Therefore, based on the schedule, which gives the time and location in which a task will be performed, the system uses the context model to predict the network bandwidth at a specific location.

\subsection{Connectivity Model}

The system's connectivity model contains a map of the network connectivity in the various known locations shown as an interval of available bandwidth for each location.

\subsection{Library of Cases}

Case-based reasoning (CBR) binds user model, task model and connectivity model together. It is possible to create a library of cases based on the task and the type of location of homecare workers. Figure 5 shows the way a case is constructed. In Figure 5, the User description represents the stereotype user model, Task represents the task model and Connection interval represents the connection model. The cases are indexed by task-ID ( $\mathrm{t} 4 \mathrm{in}$ Figure 5) and the bandwidth necessary to achieve the task.

Case 1:User description: nurse, physiotherapist Task: (t4) retrieve the patient's personal information Connection interval: $[100,375]$

Result: load the address and the name of the patient

Figure 5: An Example of Case
The process of pre-fetching consists of predicting network connectivity using the connectivity model and the user's schedule (time, location and task) and pre-fetching/hoarding the needed information using the user's task model. Figure 6 shows this process of pre-fetching information. The current location of the user and the current time is used to find the next appointment from the user's schedule (step 1). Knowing the current location and the next location, the bandwidth is retrieved from the connectivity model (step2). When the task is known from the schedule, the necessary information for the completion of the task is retrieved from the task model (step 3). Having knowledge of the bandwidth and the task, the user model is checked for any specific preferences (step 4). These four steps represent the process of retrieving an appropriate case from the case library. The fifth step is to pre-fetch/hoard the necessary information. In Mobi-Timar if a user performs different tasks which are not included in her/his schedule, the system will not guarantee the availability of information in disconnected condition.

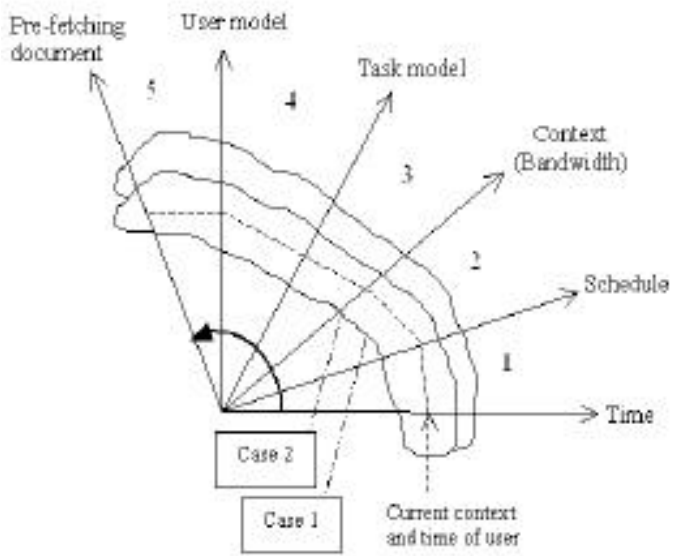

Figure 6: The Process of Pre-Fetching Information 


\section{ADAPTATION}

The proposed Mobi-Timar is based on clientserver architecture in which the agents are the middleware. The client is a Mobile Device (MD) and the server runs on a desktop computer. The server side contains the adaptation mechanism, the library of cases, the schedule, the user model, the task model, the context model, the user agents and proxy agents. The server side is encoded in Java and uses the JADE [4] platform. A proxy agent and a user agent on the server side represent each client. The client side contains a proxy agent, user agent, an application agent and an individual user model. The client side is encoded in C\#. The agents in $\mathrm{C \#}$ and Java communicate through ACL messages. Figure 7 illustrates the communication architecture.

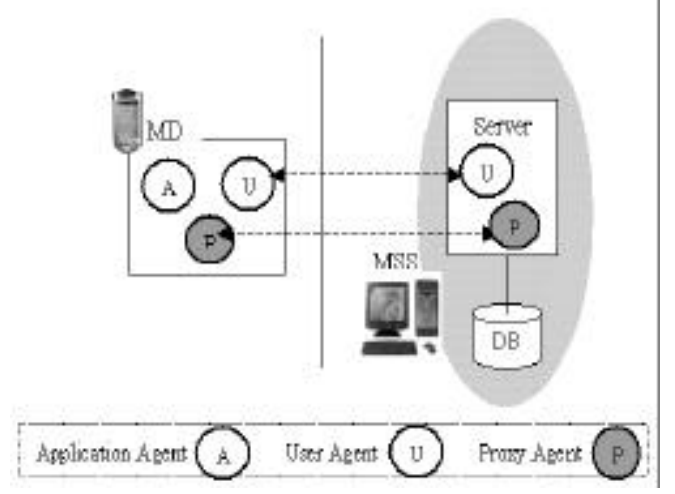

Figure 7: The Mobi-Timar Architecture

\subsection{The Use of Agents}

To give the users the impression of a seamless connection, agents are used. Agents hide changes in the network bandwidth from the user by automatically pre-fetching/hoarding the needed information from/to the server ahead of time (as shown in Figure 6).

\subsection{The Identification of Read/Write Operations}

To ensure that the read and write operations are transparent, we use two sets of agents (user agent and proxy agent) and two copies of data, one on the $\mathrm{MD}$ and the other on the server (data on the MD is a copy of the data on server). The user agent on the server side looks at any changes and updates on the server side. In the case of disconnection the user agent on the client and the server side will priorize the queue of tasks and messages that are waiting to be executed based on the user's preferences from the user model. The proxy agent of a client on the server side plays the client role during disconnection mode, while the proxy agent on the MD plays the server role during disconnection mode. The proxy agent receives the messages from server/client, waits for the connection, and then forwards the messages to the client/server. The user agent performs automatic hoarding, and the proxy agent performs automatic reintegration. The needed data for the user is cached on the MD, and the primary copy of the data is stored on the server. If the client side is disconnected and the client is executing a write operation, the client will continue with the task on MD. When the client is finished with the task on MD, the agents will wait for the connection and then execute the task on the server side and let the user know the result of the task. The status of the network bandwidth is also available for the user, so the user knows the accuracy of the data that she is using.

There are two different kinds of read and write operations, one that needs a lock and one that does not need a lock. When a user reads/writes to the schedule or the agent is pre-fetching/updating the schedule, the server side will lock the schedule to avoid any inconsistencies, such as the loss of information updates. If the client gets disconnected while reading the schedule, the schedule lock will be broken after a certain amount of time. If the client stays connected or only has a brief disconnection, the schedule stays locked until the client finishes the transaction. For agents/clients that are writing to the schedule, the schedule is locked and will not be updated until the transaction is committed. In case of an abort, the client on the MD will be notified.

Access to patient records does not require locking since information cannot be deleted or modified by homecare workers, who can only add more data. But adding the new information to the existing information cannot change the old content due to SDH policy, which states that all transactions related to homecare workers and patients should be recorded and archived. This is illustrated in Figure 8. After visiting a Patient at time $t 1$, the Physiotherapist adds information to the Patient's record using her mobile device in an area of poor connection. Some time later at time $t 2$ in an area of good connection, a Nurse adds information to the Patient's record. Her transaction is committed on the server side fairly quickly at time $t 3$. Therefore, at a later time, $t 4$, when the Physiotherapist's transaction is finally committed on the server side, the additions to the Patient's record will be ordered based on the time when each transaction was originally performed on the client's mobile device. For this reason the clocks of all mobile devices are synchronized with server's clock. 


\begin{tabular}{|c|c|c|c|c|}
\hline & \multicolumn{2}{|c|}{$\begin{array}{c}\text { Transaction committed } \\
\text { on client side } \\
\text { (On MD) }\end{array}$} & \multicolumn{2}{|c|}{$\begin{array}{c}\text { Transaction committed } \\
\text { on server side } \\
\text { (On MSS) }\end{array}$} \\
\hline & \multicolumn{4}{|c|}{ Time } \\
\hline & $\mathrm{T} 1$ & $\mathrm{t} 2$ & $\mathrm{t} 3$ & t4 \\
\hline Nurse & & $\begin{array}{l}\text { Write to } \\
\text { Patient A }\end{array}$ & $\begin{array}{l}\text { Write to } \\
\text { Patient A }\end{array}$ & \\
\hline $\begin{array}{l}\text { Physio- } \\
\text { therapist }\end{array}$ & $\begin{array}{l}\text { Write to } \\
\text { Patient A }\end{array}$ & & & $\begin{array}{l}\text { Write to } \\
\text { Patient A }\end{array}$ \\
\hline
\end{tabular}

Figure 8: An Example for Second Type of Read/Write

\subsection{Providing Only Necessary Functionalities}

The user agent can predict the next type of information access to be performed by the homecare worker using the task model (known from the user class), the connection model, the schedule and a library of cases. Based on the screen size limitation of mobile devices, the user agent provides only the suitable functionalities for the homecare worker's current access to information and not the ful functionality of Mobi-Timar since it knows which operations are going to be performed. This allows a consistent way to design the user interface around specific user tasks.

\section{EVALUATION}

The evaluation of the Mobi-Timar architecture is done using a simulation environment for the location and available bandwidth. This simulation depicts a virtual world that provides the location and the network bandwidth. The movement of each user with her MD is represented with a moving avatar in the virtual world. Each user has a pre-assigned schedule, which instructs her to move to certain locations (visit patients) and perform certain tasks. The user moves her avatar in the simulated location and performs the tasks requiring access to the patient data and schedule on her MD.

Figure 9 illustrates one of the network coverage models for this simulation. Since it is important to test the ability of Mobi-Timar to adapt quickly to drastic changes in network coverage, different models are considered, which allows for a more realistic discontinuous pattern of coverage.

The simulation shows the movement of the user through different network areas, which allows testing the transparent read and write operations and the availability of data access regardless of the location and the network bandwidth. By testing these two aspects, we will be able to evaluate the functionality of Mobi-Timar.

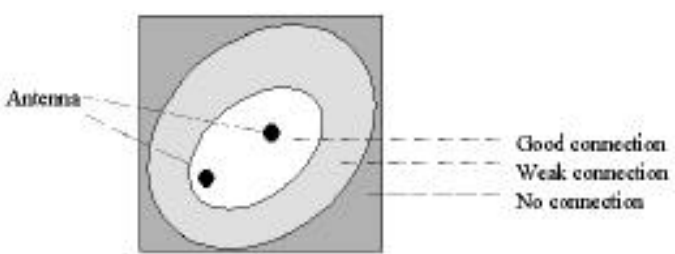

Figure 9: Different Network Coverage in the Simulation GUI

The evaluation of Mobi-Timar will be conducted by running experiments involving a centralized database containing information for four patients. Two graduate students will take the roles of a nurse and a physiotherapist. Each homecare worker will perform either a full-day or a half-day schedule, which includes all six types of operations: downloading files, updating and uploading files, sending messages, receiving messages, reading schedules, making or changing appointments. All these operations will be performed in two different conditions: connected and disconnected mode. This will allow us to evaluate the process of pre-fetching information based on the available bandwidth, accessing information during disconnection, reintegrating information and maintaining the consistency of data and user preferences on both client and server side.

The evaluation procedure involves the following two steps:

- Analyzing log data drawn from running the MobiTimar in several different bandwidth simulation models. The log file contains the homecare workers and agents transactions plus all the information transferred between the clients' devices and the server with time stamp allowing the computation of the speed of task execution.

- Using interviews to perform a usability study based on the following criteria:

- Ease of use

- User satisfaction

- Interface design and the availability of information and tasks

The evaluation process will enable us to determine the number of failures and successful $\mathrm{read} /$ write operations. It will also be possible to deduce how well the system performs when the design assumptions made earlier are valid. We will also be able to ascertain how transparent the $\mathrm{read} / \mathrm{write}$ operations were by looking at the consistency of data on both client and server side and the numbers of failed and successful operations. 


\section{RELATED WORK}

There is a great deal of research on adaptation of the application and web infrastructure for mobile devices. Most of this research, for example CRUMPET (Poslad et al., 2001a; Poslad et al., 2001b), MyCampus (Sadeh et al., 2002) and Hippie (Oppermann et al., 1999; Specht and Oppermann, 1999), focuses on content adaptation, information retrieval, interface adaptation and representation adaptation. Furthermore, the focus of most of this research is on adaptation based on the user type and the level of experience of the user. In contrast, MobiTimar adapts also based on context (e.g. current time, user task, and connection availability). Projects, such as Broad-Car (Console et al., 2002), propose adaptation based on the context of the user and use multimedia output, e.g. voice, video or text, for presenting the data to the user. In these studies, parameters used for adaptation are bandwidth, user type, task, screen size, output channels of the device, and the location. However, there are no write transactions performed in any of these systems, and synchronization and transaction management are not needed. Unlike previous approaches, as in CRUMPET, Hippie and MyCampus, our MobiTimar addresses the problem of disconnection. Agents are used to pre-fetch information relevant to the user task and context. This research doesn't focus on just-in-time retrieval; instead, it aims to support seamless access to information and modification in a timely fashion.

\section{CONCLUSIONS}

In this paper we described Mobi-Timar, an architecture for a mobile communication and information system for homecare workers, which uses a variety of techniques including user modeling, task modeling, case-based adaptation, multi-agent systems and mobile transaction management.

The result of this research is an application and a middleware for a mobile environment that can be used in other domains with similar characteristics, for example, supporting mobile technicians, salespeople, real estate agents, etc. The application ensures seamless access to data by using mobile transaction management performed by the user agent and the proxy agent. The user model, task model and context model allow the agents to select and prefetch relevant information needed for the user's next task and to create the illusion of seamless connection for the users even when the bandwidth is low or the user is disconnected.
Acknowledgement. This research has been supported by TR-Labs.

\section{REFERENCES}

CDPD (Cellular Digital Packet Data), http://www2.picante.com:81/ gtaylor/cdpd.html

Compaq iPaq, http://athome.compaq.com/showroom/static/ipaq/hand held_jumppage.asp

Console, L., Gioria, S., Lombardi, I., Surano, V., Torre, I., 2002. Adaptation and Personalization on Board Cars: A Framework and Its Application to Tourist Services. In 2nd International Conference on Adaptive Hypermedia and Adaptive Web Based Systems, AH 2002, 112-121.

JADE: http://sharon.cselt.it/projects/jade/

Jennings, N., Sycara, K. and Wooldridge, M., 1998. A RoadMap of Agent Research and Development. Journal on Autonomous Agents and Multi-Agent Systems, 1, 275-306.

Oppermann, R., Specht, M., Jaceniak, I., 1999. Hippie: A Nomadic Information System. Gellersen. W. (Ed.): Proceedings of the First International Symposium Handheld and Ubiquitous Computing (HUC'99), Karlsruhe, September 27 - 29, 330 - 333.

Pinelle, D. and Gutwin, C., 2001. Collaboration Requirements for Home Care. Research Report, University of Saskatchewan, http://hci.usask.ca/publications/index.xml

Poslad S., Laamanen H., Malaka R., Nick A., Buckle P. and Zipf, A., 2001a. CRUMPET: Creation of Userfriendly Mobile Services Personalised for Tourism. Proceedings of: $3 G 2001$ - Second International Conference on $3 G$ Mobile Communication Technologies. London, UK, 26-29.

Poslad S., Charlton P. In Marik V., Stepankova O., 2001 b. Standardizing agent interoperability: the FIPA approach. Proceedings of the Multi-Agent Systems \& Applications Conference, ACAI-01 (invited paper).

Sadeh, N., Chan, E., Shimazaki, Y., Van, 2002. MyCampus: An Agent-Based Environment for Context-Aware Mobile Services. In AAMASO2 Workshop on Ubiquitous Agents on Embedded, Wearable, and Mobile Devices, Bologna, Italy.

Specht, M., and Oppermann, R., 1999. User Modeling and Adaptivity in Nomadic Information Systems. Proceedings of the 7. GI-Workshop Adaptivität und Benutzermodellierung in Interaktiven Softwaresystemen (ABIS99), Universität Magdeburg, September 27 - October 1, 325 -328.

Vassileva, J., 1996. A Task-Centered Approach for User Modeling in a Hypermedia Office Documentation System. User Modeling and User Adapted Interaction, 6, 2-3, 185-223.

Weiser, M., 1991. The computer for the twenty-first century. Scientific American, September, 94-100. 